\title{
Transition to online teaching and learning for clerkship activities during COVID-19 in Malaysia
}

\author{
A. Sha'aban, B. Ibrahim, O. Albitar, S.G. Mohiuddin, C.G. Omar, S.N. Harun* \\ School of Pharmaceutical Sciences, Universiti Sains Malaysia, Malaysia
}

\author{
Keywords \\ Clerkship \\ COVID-19 \\ Face-to-face \\ Online Teaching \\ Learning \\ Malaysia
}

${ }^{*}$ Corresponding author:

sabariahnoor@usm.my
Summary: Prior-to the COVID-19 outbreak, undergraduate PharmCare II Clerkship activities consisted of two phases. The first phase requires pharmacy students to clerk infectious disease and endocrine-related cases of patients admitted into wards at a teaching hospital, whilst in the second phase, the students are required to present the clerked cases in front of their group members with a thorough assessment by lecturers at Universiti Sains Malaysia. Due to sudden outbreak of COVID-19, presentations at hospitals and face-to-face learning were no longer feasible; therefore an online methodology was executed. Each student was assigned a real completed case clerked by a previous Master's in Clinical Pharmacy graduate. The students then critically evaluated any pharmaceutical care issues (PCls) in each of the cases, recorded a video presentation for assessment by the lecturers and discussed each case with other group members in a synchronous online session via web-conferencing software.

\section{Background and Context}

PharmCare II Clerkship is a clinical pharmacy compulsory clerkship for Universiti Sains Malaysia (USM) final year pharmacy undergraduates. Its main objective is to develop therapeutic problem-solving skills, mainly related to infectious diseases (ID) and endocrine disorder cases.

The concepts of pharmacotherapy and pharmaceutical care of these diseases, as well as the introduction to the case clerking process and documentation in the Pharmacist's Workup of Drug Therapy (PWDT) form (Cipolle, Strand, \& Morley, 1998), were taught in other courses. Their application, however, was emphasised in this clerkship.

PharmCare II clerkship lasts for two weeks (PharmCare II Clerkship Module, 2020). It is designed in a way that a total of 12 students are on the programme every week. The first phase, lasting two days, required students to clerk one ID or endocrine case admitted into wards of a selected teaching hospital. They were also expected to obtain the patient's medication history, identify the signs and symptoms, review the medication treatment in the ward, identify any pharmaceutical care issues ( $\mathrm{PCl})$, as well as recommend, modify, monitor and evaluate the pharmacotherapy of the disease. The students are required to monitor the patient's progress and actively participate in ward rounds and discussions with the appointed hospital pharmacy preceptor. During the second phase, students present the cases in front of their group members with a thorough assessment by lecturers at the USM pharmacy school. Students' basic understanding and knowledge of the disease, competency to identify and solve PCls as well as the ability to design a monitoring plan for a patient were evaluated in the clerkship. With the COVID-19 outbreak, however, a drastic change in the clerkship activities was required to help achieve these objectives.

\section{Educational Description}

The core aim of this clerkship is to ensure that the student is able to demonstrate therapeutic problem-solving skills in ID and endocrine-related cases.

To proactively adapt to restrictions and limitations during the COVID-19 outbreak without jeopardising the core aim of the clerkship, a new mechanism for the clerkship activities had to be designed. The two phases needed to be completed online. 
To achieve this, the University used four preceptors, who were actively involved in guiding and evaluating the students. Similar to the original clerkship module, every week a group of 12 students were involved in the clerkship and they set up a WhatsApp group, which included the preceptors, to permit asynchronous learning experience.

For the first phase, the reviewing of real cases by the students was able to be maintained without requiring physical attendance at the hospital. The real ID and endocrine cases were retrieved from completed PWDT forms clerked and submitted previously by graduates of the Master's in Clinical Pharmacy programme. Details of the cases were provided to the students except for the component of the $\mathrm{PCl}$ assessment, recommendations, or monitoring plan. Unlike the original activities (where a pair of students must clerk only one case), every student in the group was assigned either an ID or endocrine case uploaded at USM E-learning portal on the day of clerkship. The students were also given a time limit to access and download the case, and to record their online attendance to ensure compliance with the clerkship timetable.

A 'to-do' checklist was given to the students to guide them on how to critically evaluate, understand, and identify PCls. The checklist was as follow:

1. Understand the case-diseases pathophysiology, clinical manifestation, general treatment and the case's chronology throughout the hospitalisation;

2. Assess abnormal subjective and objective findings;

3. Identify the drug-related problems or pharmaceutical care issues;

4. Do an intervention or suggest plans to solve the drugrelated problems (DRPs)/ pharmaceutical care issues (PCls) with evidence;

5. Plan pharmacist's monitoring plan (based on efficacy, safety, and interaction).

Students were then required to prepare a PowerPoint presentation, record a video of the presentation (not exceeding 30 minutes) and upload the footage to a designated cloud storage. The group leader was required to share the cloud storage link with the preceptors by the end of the first week of the clerkship. The groups were further divided into four sub-groups, each assigned to the four preceptors to ease the activities during phase two. Each subgroup member was required to watch all videos in the new sub-group before the web conference discussion with their preceptor. In phase two of the online clerkship, the respective sub-groups discussed the cases. Preceptors prepared at least ten questions per case which were asked at random during the session to assess students' comprehension of the cases in each sub-group.

Mark allocation was also updated based on this new learning method. Three components were assessed: case presentation (40\%); discussions and the question and answer session
(40\%); and the final updated slides (20\%). Previously, the grading was based on four components, which were hospital preceptor's evaluation (30\%); case-based clinical reasoning (CBCR) Part 1 (20\%); CBCR Part 2 (35\%); and final amended slides (15\%).

\section{Outcomes and Recommendations}

The objectives of the clerkship were fulfilled; however, only retrospective case clerking skills were able to be assessed and discussion with other healthcare personnel was not possible. Such skills, however, were developed during earlier clerkship, and problem-based solving and technology-aided learning skills were enhanced during the programme. Students' feedback on their perception of content plus general satisfaction on the new transition was received using a survey (Strachota, 2006). In terms of the students-content interaction and whether the cases used facilitated their learning, from the 56 survey responses received, $50.0 \%$ strongly agreed; $41.1 \%$ agreed; and $3.6 \%$ disagreed. Surprisingly, $41.1 \%$ and $51.8 \%$ strongly agreed and agreed, respectively that the learning activities in this clerkship required the application of problem-solving skills, which facilitated their learning. Fifty-one point eight percent $(51.8 \%)$ of students agreed that the learning activities in this clerkship required critical thinking which facilitated their learning. In terms of general satisfaction to the change from face-to-face to online learning, $41.1 \%$ were indifferent to the change; $30.4 \%$ not satisfied, while $12.5 \%$ satisfied. Just over $37.5 \%$ agreed that the online learning met their learning needs; but $37.5 \%$ did not recommend future use of the online clerkship. Moreover, $37.5 \%$ claimed not to have learnt as much in the online mode as compared to face-to-face teaching activities. Most of the students commented that they prefer to have an interactive session with patients as well as other face-to-face interaction with healthcare personnel. Most students thought that face-to-face learning would be more productive in imparting the requisite skills from experiential learning.

The end of the pandemic situation is uncertain. Thus, adapting to a new norm of teaching activities is necessary, and new methods should be improved. More ways of presenting cases could be implemented, with more time allocated to the interactive sessions with preceptors. Lumen5 or Powtoon could be used as tools, alongside PowerPoint presentations.

\section{References}

Cipolle R.J, Strand L.M., \& Morley, P.C. (1998), Pharmaceutical Care Practice. McGraw Hill, USA.

PharmCare II Clerkship Module. (2020). Universiti Sains Malaysia, Malaysia. Available at: http://farmasiklinikal.usm.my

Strachota, E. (2006). The use of survey research to measure student satisfaction in online courses. Paper presented at the Midwest Research to Practice Conference in Adult Continuing and Community Education, University of Missouri-St, USA. 